\title{
Displaced vertices as probes of sterile neutrino mixing at the LHC
}

\author{
Giovanna Cottin* \\ Department of Physics, National Taiwan University, Taipei 10617, Taiwan \\ Juan Carlos Helo ${ }^{\dagger}$ \\ Departamento de Física, Facultad de Ciencias, Universidad de La Serena, \\ Avenida Cisternas 1200, La Serena, Chile \\ and Centro-Científico-Tecnológico de Valparaíso, Casilla 110-V, Valparaíso, Chile \\ Martin Hirsch \\ AHEP Group, Instituto de Física Corpuscular-CSIC/Universitat de València, \\ Edificio de Institutos de Paterna, Apartado 22085, E-46071 València, Spain
}

(Received 25 June 2018; published 8 August 2018)

\begin{abstract}
We investigate the reach at the LHC to probe light sterile neutrinos with displaced vertices. We focus on sterile neutrinos $N$ with masses $m_{N} \sim(5-30) \mathrm{GeV}$ that are produced in rare decays of the standard model gauge bosons and decay inside the inner trackers of the LHC detectors. With a strategy that triggers on the prompt lepton accompanying the $N$ displaced vertex and considers charged tracks associated with it, we show that the $13 \mathrm{TeV}$ LHC with $3000 / \mathrm{fb}$ is able to probe active-sterile neutrino mixings down to $\left|V_{l N}\right|^{2} \approx 10^{-9}$, with $l=e, \mu$, which is an improvement of up to 4 orders of magnitude when comparing with current experimental limits from trileptons and proposed lepton-jets searches. In the case when $\tau$ mixing is present, mixing angles as low as $\left|V_{\tau N}\right|^{2} \approx 10^{-8}$ can be accessed.
\end{abstract}

DOI: 10.1103/PhysRevD.98.035012

\section{INTRODUCTION}

Searches for new physics responsible for the lightness of neutrino masses [1] have been in the program of the LHC experiments for decades [2]. This new physics beyond the standard model (SM) may be explained by the so-called see-saw mechanism [3] that introduces the existence of heavy right-handed (sterile) neutrinos that mix with the neutrinos in the standard model [4,5]. For low-enough mixing angles and sterile neutrino masses below the electroweak scale, the sterile neutrino $N$ can be long-lived and may decay with a characteristic displaced vertex (DV) signature inside particle detectors.

Experimental efforts to search for these states at hadron colliders normally focus on promptly decaying sterile neutrinos with masses $m_{N} \sim \mathcal{O}(100) \mathrm{GeV}$. Earlier searches by ATLAS [6] and CMS [7,8] focus on the Majorana signature of same-sign dileptons and jets [9]. Only recently, the CMS experiment has provided limits for $N$ masses below

\footnotetext{
*gcottin@phys.ntu.edu.tw

jchelo@userena.cl

mahirsch@ific.uv.es
}

Published by the American Physical Society under the terms of the Creative Commons Attribution 4.0 International license. Further distribution of this work must maintain attribution to the author(s) and the published article's title, journal citation, and DOI. Funded by SCOAP ${ }^{3}$.
$40 \mathrm{GeV}$ in the search for three prompt charged leptons [10]. For current bounds on sterile neutrino mixing, see [11,12]. Attention to displaced vertex signatures, which can probe masses in the $\mathrm{GeV}$ range, is rapidly growing. Recent phenomenological studies assessing the LHC sensitivity with displaced vertices to light sterile neutrinos in various models are studied in [13-18]. Despite the technical challenges in the reconstruction and modeling of the detector response to displaced vertices, this is an important signal of new physics as it is scarce in the standard model and has to be explored further in order to ensure the successful exploration of new physics at the LHC and across all mass ranges.

In this work, we use a strategy motivated by the ATLAS multitrack displaced vertex search $[19,20]$, recently validated in our previous work [21] in the context of a left-right symmetric model. Here we focus on a simplified model in which the standard model field content is extended by one heavy sterile neutrino, briefly described in Sec. II. We consider three cases for active-sterile neutrino mixing $V_{l N}$, for each of the active flavors $l=e, \mu, \tau$. The displaced vertex strategy implemented in each case is described in Sec. III. Discovery prospects and reach at the LHC are discussed in Sec. IV. We close the paper and summarize in Sec. V.

\section{STERILE NEUTRINO SIMPLIFIED MODEL}

We consider a simplified see-saw model of neutrinos based on the standard model gauge group, in which only 


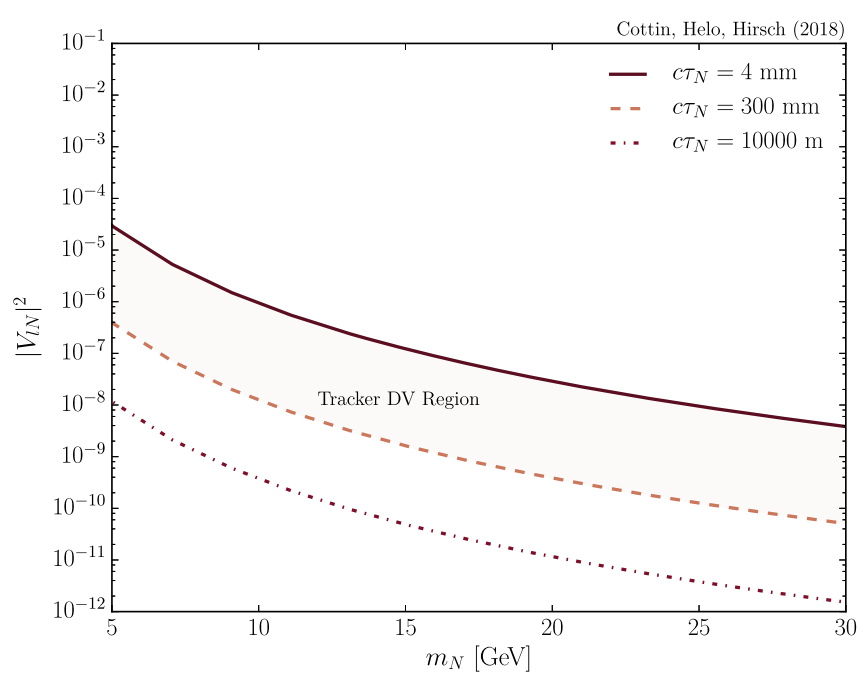

FIG. 1. Contours of fixed sterile neutrino proper decay distance $c \tau_{N}$ as a function of mass and active-sterile neutrino mixing angle. The shaded region represents roughly the region that can be accessed with current displaced vertex searches in the ATLAS inner tracker $[19,20]$.

one massive sterile neutrino $N$ is present in the kinematic range of our interest. In this generic framework, $N$ couples to the SM leptons via a small mixing in the electroweak currents. The charged and neutral current interactions of this model are described in [22].

We consider one neutrino flavor at a time, $e, \mu$ or $\tau$, produced in $W$ boson decays in association with the respective lepton flavor: $W^{ \pm} \rightarrow N l^{ \pm}$. The $N$ decays proceed via $N \rightarrow l^{ \pm} q \bar{q}, N \rightarrow l^{\prime \mp} l^{ \pm} \nu_{l}$ and $N \rightarrow \nu_{l} q \bar{q}$. The $N$ proper lifetime is given by [22]

$$
c \tau_{N} \sim 3.7\left(\frac{1 \mathrm{GeV}}{m_{N}}\right)^{5}\left(\frac{0.1}{\left|V_{l N}\right|^{2}}\right)[\mathrm{mm}]
$$

The relevant parameters are the sterile neutrino mass $m_{N}$ and active-sterile neutrino mixing $\left|V_{I N}\right|^{2}$, which we treat as independent. In general, small neutrino masses and mixing lead to macroscopic lifetimes. In principle one can have a large $N$ lifetime by making the mixing very small (instead of the sterile neutrino mass). However, for smaller values of the mixing, the production rate of $N$ also becomes smaller. We are interested in masses in the $\mathrm{GeV}$ range to access lifetimes of the order of picoseconds while scanning over mixings as low as $\left|V_{l N}\right|^{2}=10^{-12}$.

In Fig. 1 we show different values of the proper lifetime in the $\left(m_{N},\left|V_{l N}\right|^{2}\right)$ plane. We highlight the approximated region where vertices can efficiently be reconstructed inside the tracker region of ATLAS, for proper decay distances between 4 and $300 \mathrm{~mm}$. We are interested in sterile neutrino mass between $5 \mathrm{GeV}<m_{N}<30 \mathrm{GeV}$, which can be probed with a multitrack displaced vertex search at ATLAS or CMS.

\section{SIMULATIONS AND SELECTION OF DISPLACED EVENTS}

We generate a UFO [23] model with SARAH [24] and use SPHENO $[25,26]$ for the spectrum calculation of the sterile neutrino simplified model. We simulate events at $\sqrt{s}=$ $13 \mathrm{TeV}$ for the process $p p \rightarrow W^{ \pm} \rightarrow N l^{ \pm}$. Generation is performed with MADGRAPH5_AMC@NLOv2.4.3 [27] at leading order. We normalize the corresponding value to match the experimental cross section in Ref. [28]. The generated events are then interfaced to PYTHIA8 V2.3 [29] for showering, hadronization and computation of the $N$ decays. Plots are generated with matplotlib [30].

The promising decay channels for the sterile neutrino are semileptonically $N \rightarrow l^{ \pm} q \bar{q}$ and leptonically $N \rightarrow l^{\prime \mp} l^{ \pm} \nu_{l}$. Decays via a neutral current such that $N \rightarrow \nu_{l} q \bar{q}$ are also possible [22]. All these modes will lead to displaced vertices with charged tracks associated with them. For the case when only $\tau$ mixing is present, both semileptonic and leptonic decays lead to the presence of a $\tau$ lepton coming from the displaced vertex.

We propose a search inspired by the ATLAS multitrack displaced vertex analysis $[19,20]$, which is sensitive to lifetimes of the order of picoseconds to about a nanosecond, so particle decays can be reconstructed with a displaced vertex signature inside the inner tracker. This strategy was developed in our recent work in Ref. [21], where we trigger on the prompt lepton coming from the $W$ boson decay, impose cuts on the neutrino displaced vertex and its decay products, and apply vertex-level efficiencies (made public by ATLAS in [19]) to DVs that pass the required particle-level acceptance cuts. Since the parametrized selection efficiencies provided by ATLAS assumes all decay products are prompt from the DV, they are not directly applicable to the case when there is a $\tau$ lepton coming from the displaced vertex, ${ }^{1}$ so we consider decays to $N \rightarrow \nu_{l} q \bar{q}$ only when $\tau$ mixing is present.

Prompt leptons are reconstructed considering the following:

For electrons: We require an isolated electron within $|\eta|<2.5$. We smear their momenta with a resolution of $2 \%$ at $10 \mathrm{GeV}$, falling linearly to $1 \%$ at $100 \mathrm{GeV}$, and then $1 \%$ flat.

For muons: We require an isolated muon within $|\eta|<2.5$. We smear their momenta with a resolution between $|\eta|$ of 2 and 0 , linearly falling from $4 \%$ to $1.5 \%$.

\footnotetext{
${ }^{1}$ The further displacement of $\tau$ 's inside the vertex will affect the vertex reconstruction efficiency from the subsequent displacement of taus (and from any heavy flavor quark in general). This was addressed for example in Ref. [31], when there are two $b^{\prime}$ s coming from the displaced vertex. By allowing a bigger merging distance of $5 \mathrm{~mm}$ (instead of $1 \mathrm{~mm}$ ) when forming a vertex, some efficiency is recovered. Since we do not implement a vertex reconstruction algorithm in this work, the loss in efficiency can not be estimated.
} 
For taus: We implement a basic reconstruction following Ref. [32]. We start by reconstructing jets with FASTJET 3.1.3 [33] using the anti- $k_{t}$ algorithm with distance parameter $R=0.4$. Only jets with $p_{T}>10 \mathrm{GeV}$ and $|\eta|<2.5$ are taken as seeds for $\tau$ reconstruction. Charged constituents inside the jet must have $p_{T}>1 \mathrm{GeV}$. If a truth $\tau$ candidate falls within a cone $\Delta R<0.2$ centered on the jet axis, it is selected.

The following selections are then imposed:

(1) One prompt lepton (as reconstructed above) with $p_{T}>25 \mathrm{GeV}$.

(2) Decay position of the DV contained within transverse distance $r_{\mathrm{DV}}<300 \mathrm{~mm}$, and $\left|z_{\mathrm{DV}}\right|<300 \mathrm{~mm}$. The distance between the interaction point and the decay position must be bigger than $>4 \mathrm{~mm}$.

(3) Decay products must be charged (i.e., tracks) with $p_{T}>1 \mathrm{GeV}$ and transverse impact parameter $\left|d_{0}\right|>2 \mathrm{~mm} . d_{0}$ is defined as $d_{0}=r_{D V} \times \sin \Delta \phi$, with $\Delta \phi$ being the azimuthal angle between the decay product and the trajectory of the long-lived $N$.

(4) The number of selected tracks $N_{\text {trk }}$ must be at least 3. The invariant mass of the DV $m_{\mathrm{DV}}$ must be $\geq 5 \mathrm{GeV}$, and assumes all tracks have the mass of the pion.

(5) Parametrized selection efficiencies are applied depending on the displaced vertex distance (within 4 and $300 \mathrm{~mm}$, between the pixel and the SCT), number of tracks and mass.

As pointed out in [21], with these selections we are still in a zero background region, where background comes mostly from instrumental sources.

\section{SENSITIVITY REACH}

We analyze the region where a displaced search with the above selections can have sensitivity. The relevant parameters in the sterile neutrino model are the neutrino mass $m_{N}$ and active-sterile neutrino mixing $\left|V_{l N}\right|^{2}$.

We first chose a representative benchmark point with $m_{N}=15 \mathrm{GeV},\left|V_{l N}\right|^{2}=10^{-8}$ and proper neutrino decay distance $c \tau_{N} \approx 50 \mathrm{~mm}$ to illustrate the effect of all analysis cuts from the previous section. We combine the second and third cuts in the "DV fiducial" entry in Table I. Events are generated at $13 \mathrm{TeV}$ which corresponds to a production cross section of $0.09 \mathrm{fb}$. Cut-flows in the case of electron, muon and tau mixing are shown.

As already noted in the context of a left-right symmetric model in [21], for sterile neutrinos with masses below the electroweak scale this search strategy looses sensitivity, as lower masses will lead to softer decay products with limited amount of tracks available to make up a vertex. However, given the low background nature of this signature, the discovery of a displaced vertex signal in the sterile neutrino model is possible at the high-luminosity LHC.

Figures 2-4 show the estimated reach in the $\left(m_{N},\left|V_{l N}\right|^{2}\right)$ plane, in the case of electron, muon and tau mixing,
TABLE I. Numbers of simulated events at $\sqrt{s}=13 \mathrm{TeV}$ for a benchmark with $m_{N}=15 \mathrm{GeV}, \quad\left|V_{l N}\right|^{2}=10^{-8}$, and $c \tau_{N} \approx 50 \mathrm{~mm}$, in the three cases considered where $l=e, \mu$ or $\tau$. Overall efficiencies $\epsilon$ after all cuts are also shown.

\begin{tabular}{lccr}
\hline \hline & $e$ mixing & $\mu$ mixing & $\tau$ mixing \\
\cline { 2 - 4 } & $\epsilon=4.88 \%$ & $\epsilon=4.21 \%$ & $\epsilon=0.7 \%$ \\
\hline All events & 10000 & 10000 & 10000 \\
Prompt $l$ & 4004 & 3321 & 764 \\
DV fiducial & 2266 & 2058 & 433 \\
$N_{\text {trk }}$ & 814 & 712 & 177 \\
$m_{\text {DV }}$ & 645 & 579 & 72 \\
DV efficiency & 488 & 421 & 70 \\
\hline \hline
\end{tabular}

respectively. We also show for comparison the projected sensitivities from the proposed SHiP [34] and MATHUSLA $[35,36]$ experiments. For projected sensitivities for other proposals for heavy neutral leptons within $m_{N}<5 \mathrm{GeV}$, see FASER [14,37,38] and CODEX-b [14,39].

In Fig. 2, we see that mixings as low as $\left|V_{e N}\right|^{2} \approx 1.5 \times$ $10^{-9}$ for $3000 / \mathrm{fb}$ and $13 \mathrm{TeV}$ can be probed for $m_{N}=20 \mathrm{GeV}$. Current neutrinoless double beta decay $(0 \nu \beta \beta)$ experiments and searches for trileptons at the LHC are also sensitive to $\mathcal{O}(10) \mathrm{GeV}$ sterile neutrino masses. We show an update of the limits calculated in [22] using the latest limit on $0 \nu \beta \beta$ from the GERDA experiment [43]. LHC limits for sterile neutrino masses

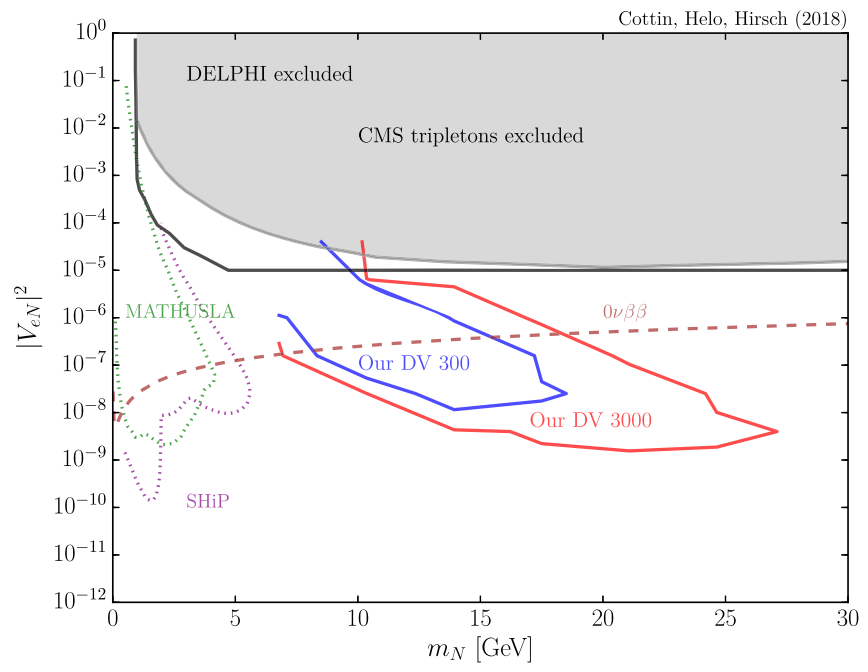

FIG. 2. $95 \% \mathrm{CL}$ reach in the $\left(\left|V_{e N}\right|^{2}, m_{N}\right)$ plane at $\sqrt{s}=$ $13 \mathrm{TeV}$ of our proposed multitrack displaced strategy for $\mathcal{L}=$ $300 \mathrm{fb}^{-1}$ (blue) and $\mathcal{L}=3000 \mathrm{fb}^{-1}$ (red). Projected sensitivities for the MATHUSLA (dashed green) and SHiP (dashed purple) experiments are also shown for comparison (taken from Refs. [14,40], respectively). The DELPHI limit, taken from [2], is also shown. A limit derived from $0 \nu \beta \beta$ experiments is also shown (see text for more details). The 95\% CL exclusion of the CMS $13 \mathrm{TeV}$ trileptons search [10] is given by the filled grey region. 


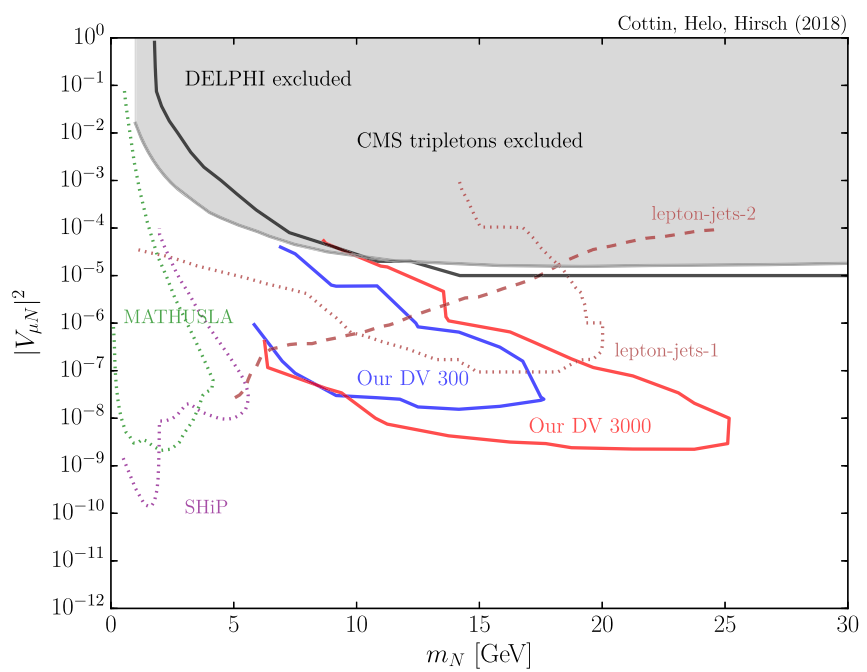

FIG. 3. $95 \% \mathrm{CL}$ reach in the $\left(\left|V_{\mu N}\right|^{2}, m_{N}\right)$ plane at $\sqrt{s}=$ $13 \mathrm{TeV}$ of our proposed multitrack displaced strategy for $\mathcal{L}=$ $300 \mathrm{fb}^{-1}$ (blue) and $\mathcal{L}=3000 \mathrm{fb}^{-1}$ (red). Projected sensitivities for the MATHUSLA (dashed green) and SHiP (dashed purple) experiments are also shown for comparison (taken from Refs. [14,40], respectively). The DELPHI limit, taken from [2], is also shown. The $13 \mathrm{TeV}$ limit from proposed lepton-jets searches are shown in dashed brown, taken from Ref. [41] ("lepton-jets1") and Ref. [42] ("lepton-jets2"). See the text for more details. The 95\% CL exclusion of the CMS $13 \mathrm{TeV}$ trileptons search [10] is given by the filled grey region.

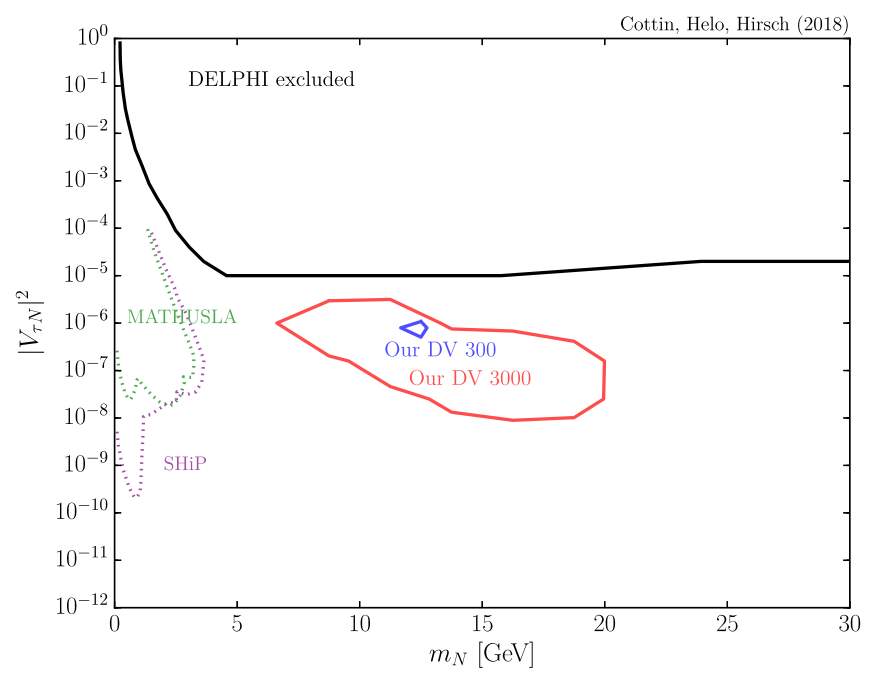

FIG. 4. $95 \% \mathrm{CL}$ reach in the $\left(\left|V_{\tau N}\right|^{2}, m_{N}\right)$ plane at $\sqrt{s}=$ $13 \mathrm{TeV}$ of our proposed multitrack displaced strategy for $\mathcal{L}=$ $300 \mathrm{fb}^{-1}$ (blue) and $\mathcal{L}=3000 \mathrm{fb}^{-1}$ (red). Projected sensitivity the MATHUSLA (dashed green) and for the SHiP (dashed purple) experiment is also shown for comparison (taken from Ref. [35]). The DELPHI limit, taken from [2], is also shown for comparison. below $40 \mathrm{GeV}$ are presented for the first time in the CMS $13 \mathrm{TeV}$ search for three prompt charged leptons in the final state [10]. Other significant constrain in our mass region of interest comes from LEP data, where the DELPHI collaboration provides limits on sterile states produced in decays of the $Z$ boson [44].

In Fig. 3, mixings as low as $\left|V_{\mu N}\right|^{2} \approx 2.2 \times 10^{-9}$ for $3000 / \mathrm{fb}$ and $13 \mathrm{TeV}$ can be probed for $m_{N}=20 \mathrm{GeV}$. We also show limits from proposals with lepton-jets searches, which is a complementary strategy. The curve labeled "lepton-jets-1" shows the exclusion form Ref. [41] at $13 \mathrm{TeV}$ and $300 / \mathrm{fb}$, where zero background is assumed. The curve labeled "lepton-jets-2" shows the $13 \mathrm{TeV}$ limit in [42] and $300 / \mathrm{fb}$, and considers additional background sources to the ones in [41]. An improvement of roughly 2 orders of magnitude in sensitivity is achieved with our strategy with $300 / \mathrm{fb}$ for masses $5<m_{N}<25 \mathrm{GeV}$. The 95\% CL exclusion of the CMS $13 \mathrm{TeV}$ prompt trileptons search [10] is also shown, proving to be competitive with DELPHI [44].

Finally, we show in Fig. 4 the reach when there is $\tau$ mixing. Experimental limits for $\tau$ mixing have not been addressed yet at the LHC. We show the DELPHI limit [44] from $Z$ decays for comparison. For taus, a large luminosity sample will be needed for obtaining meaningful constraints. As the figure shows, there is only a very narrow region testable with $300 / \mathrm{fb}$. Mixings as low as $\left|V_{\tau N}\right|^{2} \approx$ $8 \times 10^{-9}$ for $m_{N}=16 \mathrm{GeV}$ at $3000 / \mathrm{fb}$ can be probed with this strategy.

\section{SUMMARY AND CONCLUSIONS}

We study the potential of the LHC to probe light sterile neutrinos, and active-sterile neutrino mixing angles, with a displaced vertex strategy motivated by current multitrack DV searches at the $13 \mathrm{TeV}$ LHC. We focus on a simplified model where the standard model is extended with one sterile neutrino $N$. Mixing with the three flavors $e, \mu$ and $\tau$, are treated separately. In all cases, to our knowledge, we see that this strategy probes to be the most sensitive to date in the mass region of interest $\left(5 \mathrm{GeV}<m_{N}<30 \mathrm{GeV}\right)$.

For $e$ mixing, we show that this DV search is more sensitive than current neutrinoless double beta decay experiments. In the case of $\mu$ mixing, parts of the parameter space not accessible with other LHC searches (such as lepton-jets or trileptons) is possible. In both cases, with $3000 / \mathrm{fb}$, an improvement of up to 4 orders of magnitude $\left(\left|V_{l N}\right|^{2} \approx \times 10^{-9}\right.$ for sterile neutrino masses between 5 and $20 \mathrm{GeV}$ ) in sensitivity is gained when comparing with the current experimental limits from trileptons searches at CMS [10].

Accessing $\tau$ mixing is less straightforward due to the difficulty in reconstructing $\tau$ leptons, and also since the presence of a subsequently displaced $\tau$ coming from the displaced vertex affects the vertex reconstruction efficiency in a way that is not trivial to quantify. We access 
$\tau$ mixing by considering sterile neutrino decays via a neutral current only, which still leads to a displaced vertex formed from hadronized tracks, to which publicly available DV efficiencies can be applied, thus avoiding the problematic $\tau$ in the DV. This may be an advantage of a multitrack based strategy, as opposed to tagging leptons coming from the DV, in constraining $\tau$ mixing.

We briefly comment on prospects for testing sterile neutrinos at LHCb. The authors in [45] discuss limits for a different model than the one presented in this work. They show that sterile neutrino masses around $9 \mathrm{GeV}$ and mixings down to $\approx 10^{-6}$ can be constrained in semileptonic sterile neutrino decays $(\mu q \bar{q})$ at the $95 \%$ CL with current LHCb data [46]. Mixings up to $\approx 10^{-8}$ (for masses between $15-25 \mathrm{GeV}$ ) can be further probed at higher luminosity, suggesting limits for $\mu$ mixing from $\mathrm{LHCb}$ could be competitive in this mass region to the ones we derived here in the context of ATLAS.
Finally, the sensitivity of this displaced strategy at the LHC is complementary to that of future fixed-target experiments, such as SHiP, or the MATHUSLA surface detector, which can probe sterile neutrino masses below $5 \mathrm{GeV}$. This makes a tracker based DV search for light sterile neutrinos unique, as it has no competition from other experiments within $5 \mathrm{GeV}<m_{N}<30 \mathrm{GeV}$.

\section{ACKNOWLEDGMENTS}

G. C. acknowledges support by the Ministry of Science and Technology of Taiwan under Grant No. MOST-1062811-M-002-035. J. C. H. is supported by Chile Grants Fondecyt No. 1161463, Conicyt PIA/ACT 1406, and Basal FB0821. M. H. was funded by Spanish MICINN Grants No. FPA2017-85216-P and No. SEV-2014-0398 (from the Ministerio de Economía, Industria y Competitividad), as well as No. PROMETEOII/2014/084 (from the Generalitat Valenciana).
[1] K. A. Olive et al. (Particle Data Group), Chin. Phys. C 38, 090001 (2014).

[2] F. F. Deppisch, P. S. Bhupal Dev, and A. Pilaftsis, New J. Phys. 17, 075019 (2015).

[3] P. Minkowski, Phys. Lett. 67B, 421 (1977).

[4] R. N. Mohapatra and G. Senjanovic, Phys. Rev. Lett. 44, 912 (1980).

[5] J. Schechter and J. W. F. Valle, Phys. Rev. D 22, 2227 (1980).

[6] G. Aad et al. (ATLAS Collaboration), J. High Energy Phys. 07 (2015) 162.

[7] V. Khachatryan et al. (CMS Collaboration), Phys. Lett. B 748, 144 (2015).

[8] V. Khachatryan et al. (CMS Collaboration), Eur. Phys. J. C 74, 3149 (2014).

[9] W.-Y. Keung and G. Senjanovic, Phys. Rev. Lett. 50, 1427 (1983).

[10] A. M. Sirunyan et al. (CMS Collaboration), Phys. Rev. Lett. 120, 221801 (2018).

[11] G. Cvetič, A. Das, and J. Zamora-Saá, arXiv:1805.00070.

[12] A. Das and N. Okada, Phys. Lett. B 774, 32 (2017).

[13] F. F. Deppisch, W. Liu, and M. Mitra, arXiv:1804.04075.

[14] J. C. Helo, M. Hirsch, and Z. S. Wang, J. High Energy Phys. 07 (2018) 056.

[15] S. Jana, N. Okada, and D. Raut, arXiv:1804.06828.

[16] M. Nemevšek, F. Nesti, and G. Popara, Phys. Rev. D 97, 115018 (2018).

[17] I. Lara, D. E. López-Fogliani, C. Muñoz, N. Nagata, H. Otono, and R. Ruiz De Austri, arXiv:1804.00067.

[18] P. S. B. Dev, R. N. Mohapatra, and Y. Zhang, Nucl. Phys. B923, 179 (2017).

[19] M. Aaboud et al. (ATLAS Collaboration), Phys. Rev. D 97, 052012 (2018).
[20] G. Aad et al. (ATLAS Collaboration), Phys. Rev. D 92, 072004 (2015).

[21] G. Cottin, J. C. Helo, and M. Hirsch, Phys. Rev. D 97, 055025 (2018).

[22] J. C. Helo, M. Hirsch, and S. Kovalenko, Phys. Rev. D 89, 073005 (2014); 93, 099902 (2016).

[23] C. Degrande, C. Duhr, B. Fuks, D. Grellscheid, O. Mattelaer, and T. Reiter, Comput. Phys. Commun. 183, 1201 (2012).

[24] F. Staub, Comput. Phys. Commun. 185, 1773 (2014).

[25] W. Porod and F. Staub, Comput. Phys. Commun. 183, 2458 (2012).

[26] W. Porod, Comput. Phys. Commun. 153, 275 (2003).

[27] J. Alwall, R. Frederix, S. Frixione, V. Hirschi, F. Maltoni, O. Mattelaer, H. S. Shao, T. Stelzer, P. Torrielli, and M. Zaro, J. High Energy Phys. 07 (2014) 079.

[28] G. Aad et al. (ATLAS Collaboration), Phys. Lett. B 759, 601 (2016).

[29] T. Sjöstrand, S. Ask, J. R. Christiansen, R. Corke, N. Desai, P. Ilten, S. Mrenna, S. Prestel, C. O. Rasmussen, and P. Z. Skands, Comput. Phys. Commun. 191, 159 (2015).

[30] J. D. Hunter, Comput. Sci. Engineering 9, 90 (2007).

[31] B. C. Allanach, M. Badziak, G. Cottin, N. Desai, C. Hugonie, and R. Ziegler, Eur. Phys. J. C 76, 482 (2016).

[32] G. Aad et al. (ATLAS Collaboration), Eur. Phys. J. C 75, 303 (2015).

[33] M. Cacciari, G. P. Salam, and G. Soyez, Eur. Phys. J. C 72, 1896 (2012).

[34] S. Alekhin et al., Rept. Prog. Phys. 79, 124201 (2016).

[35] D. Curtin et al., arXiv:1806.07396.

[36] J. P. Chou, D. Curtin, and H. J. Lubatti, Phys. Lett. B 767, 29 (2017).

[37] J. L. Feng, I. Galon, F. Kling, and S. Trojanowski, Phys. Rev. D 97, 035001 (2018). 
[38] F. Kling and S. Trojanowski, Phys. Rev. D 97, 095016 (2018).

[39] V. V. Gligorov, S. Knapen, M. Papucci, and D. J. Robinson, Phys. Rev. D 97, 015023 (2018).

[40] K. Bondarenko, A. Boyarsky, D. Gorbunov, and O. Ruchayskiy, arXiv:1805.08567.

[41] E. Izaguirre and B. Shuve, Phys. Rev. D 91, 093010 (2015).

[42] S. Dube, D. Gadkari, and A. M. Thalapillil, Phys. Rev. D 96, 055031 (2017).
[43] M. Agostini et al. (GERDA Collaboration), Phys. Rev. Lett. 120, 132503 (2018).

[44] P. Abreu et al. (DELPHI Collaboration), Z. Phys. C 74, 57 (1997); 75, 580(E) (1997).

[45] S. Antusch, E. Cazzato, and O. Fischer, Phys. Lett. B 774, 114 (2017).

[46] R. Aaij et al. (LHCb), Eur. Phys. J. C 77, 224 (2017). 